\title{
Modification of Zenner and Liu Criterion Due to Non-Proportionality of Fatigue Load by Means of MCE Approach
}

\author{
D. Skibicki ${ }^{1}$ and L. Pejkowski ${ }^{2}$ \\ Faculty of Mechanical Engineering, University of Technology and Life Sciences in \\ Bydgoszcz, Prof. S. Kaliskiego 7, 85-796 Bydgoszcz, Poland \\ 1 e-mail: dariusz.skibicki@utp.edu.pl \\ 2 e-mail: lukasz.pejkowski@utp.edu.pl
}

\begin{abstract}
The integral approach is considered to be the proper one to be used for formulation of multi-axial fatigue criteria. The results of experimental verification of belonging to this group Zenner criterion confirm its efficiency in for nonproportional loads. Thanks to application of a generalized fatigue parameter, this criterion is useful for different types of proportional loads and for various materials. However, for non-proportional loads of high non-proportionality degree, the fatigue estimation error often exceeds scatter band 3. In this paper a proposal has been presented concerning modification of Zenner criterion. It involves introduction of a load non-proportionality measure by means of the MCE method.
\end{abstract}

\section{INTRODUCTION - THE IDEA OF INTEGRAL APPROACH}

The idea of integral approach involves determination of mean values of the fatigue damage parameters within the material elementary volume $V$. These quantities depend on normal and shear stresses exerted on the plane. Its location is described within a system of spherical coordinates $\varphi \in\langle 0,2 \pi\rangle$ i $\gamma \in\langle 0, \pi\rangle$. Double integration of these variables yields a square mean of the considered quantity. Thanks to such an approach it is possible to account for all the planes passing through a given point of the material. This kind of approach refers to interpretation of the second invariant of stress state deviator $J_{2}$, proposed by Novoshilov [1], as a square mean of shear stresses located on each of the planes passing through the material considered point.

\section{ZENNER AND LIU CRITERION IN CASE OF NON-PROPORTIONAL LOADS}

In his works [1, 2, 3], Zenner notes that Novoshilov, in his approach, accounts neither for the phase shift angle between the courses of load components nor for normal stress. This kind of approach remains inconsistent with the results of experimental tests which 
confirm an impact of the phase shift and mean stress on the material fatigue behavior. $\mathrm{HMH}$ hypothesis also assumes a constant ratio of tension-compression $\sigma_{T C}$ up to torsion fatigue limit $\tau_{T}$ equal to $1 / \sqrt{3}$. Whereas Zenner et al. [3] claim that for ductile materials this ratio is contained in interval $0.5<\left(\sigma_{T C} / \tau_{T}\right)<0.8$.

According to the above mentioned observations the fatigue criterion has been formulated, which for loads with no mean values (means are not the subject of analysis of this study), can be written as follows:

$$
\sigma_{Z}=\sqrt{\frac{15}{8 \pi} \int_{\gamma=0}^{\pi} \int_{\varphi=0}^{2 \pi}\left(a \tau_{\gamma \varphi, a^{2}}+b{\sigma_{\gamma \varphi, a^{2}}}^{2} \sin \gamma d \gamma d \varphi\right.} \leq \sigma_{T C}
$$

Coefficients $\mathrm{a}$ and $\mathrm{b}$ are dependent on the material properties. They are determined on the basis of the following formulas:

$$
a=\frac{1}{5}\left(3\left(\frac{\sigma_{T C}}{\tau_{T}}\right)^{2}-4\right), \quad b=\frac{2}{5}\left(3-\left(\frac{\sigma_{T C}}{\tau_{T}}\right)^{2}\right) .
$$

In case of non-proportional loads, as Papadopoulos et al. [4] have rightly observed, the question as to which quantity can be accepted as the amplitude of shear stress $\tau_{\gamma \varphi, a}$ is still open. According to Zenner et al. [3], it is the shear stress vector module, maximal during one load cycle, to be accepted as amplitude $\tau_{\gamma \varphi, a}$. Such an approach does not account for the fact that during a cycle of non-proportional load, apart from the module, also the vector of shear stress changes its direction, drawing a hodograph. For instance, in case of tension-compression with alternate torsion, the vector of tangent stress draws an ellipse shaped path on the plane, as in Fig.1.
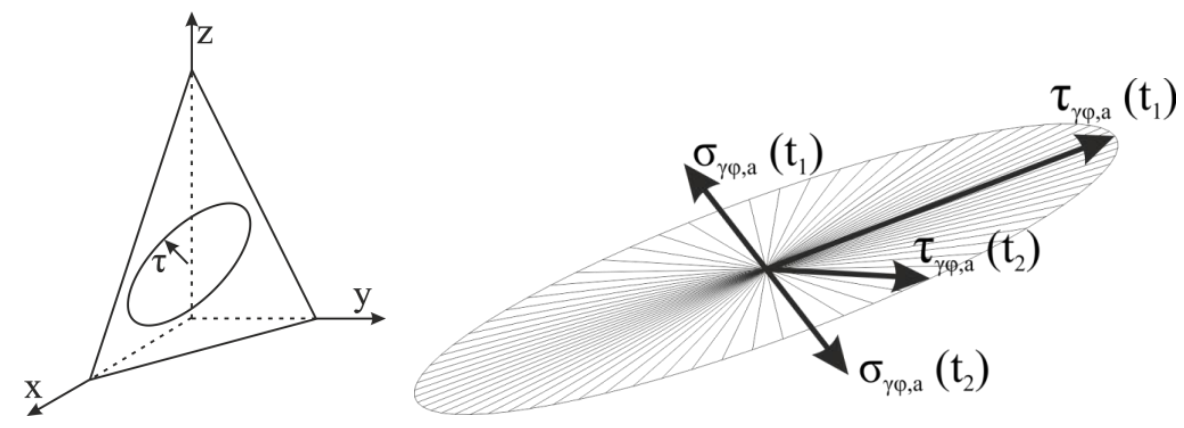

Figure 1. Changes in the module and tangent stress vector direction on the material surface during a non-proportional load cycle. 
Experimental tests [5] have shown that the direction change of shear stress vector (load path change) has a significant influence on the material fatigue behavior. Therefore, it should not be omitted while formulating fatigue criteria.

\section{A PROPOSAL OF MODIFICATION OF ZENNER AND LIU CRITERION}

The proposed modification of Zenner and Liu criterion can be divided into three steps. The first one involves an assumption that each moment of the load cycle can be considered as a separate state of non-proportional load. This assumption is based on the results of experimental verification of Zenner and Liu criterion which confirms its usefulness for assessment of fatigue life of materials exposed to uniaxial and complex proportional loads [6]. The value of equivalent stress $\sigma_{Z}$ according to Eq. 8 is calculated for each of these moments. The maximal value of $\sigma_{Z}$ in time, denoted as $\sigma_{Z, \max (t)}$ is accepted as the quantity representing the whole cycle of load. Fig. 2 shows a time course of equivalent stresses calculated in the above described manner.

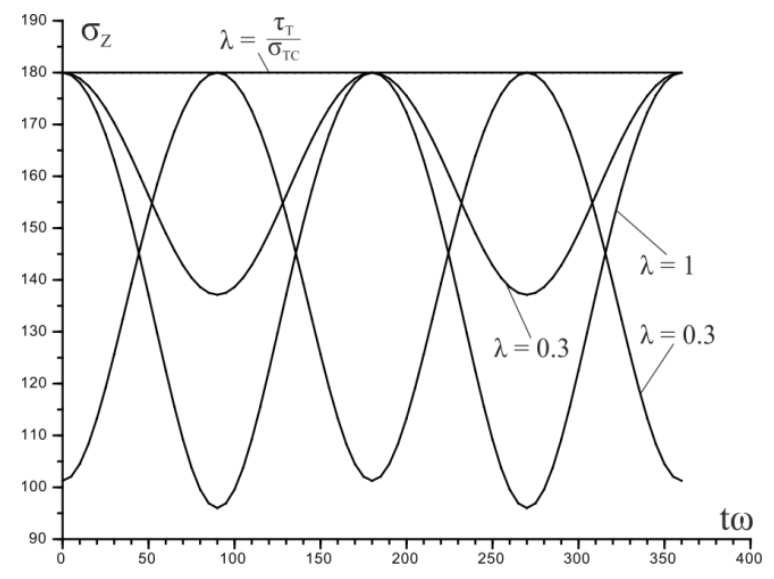

Figure 2. Equivalent stress according to Zenner and Liu criterion for CuETP, calculated for each moment of non-proportional load cycle with phase shift equal to $\delta=90^{\circ}$.

For proportional loads, such an approach yields a result identical to the unmodified criterion. Whereas in case of non-proportional loads the unmodified criterion provides underestimated values of equivalent stresses. In Fig. 3 there are S-N curves obtained on the basis of equivalent stresses according to Zenner and Liu criterion for CuETP. The curves have been determined for uniaxial tension-compression (T-C), alternate torsion, complex proportional load with $\lambda$ equal to 0.5 (P05), and non-proportional loads with phase shift $\delta=90^{\circ}$, and with coefficients $\lambda$ equal to 0.3 (N03), 0.5 (N05), 0.7 (N07) and 0.8 (N08). Curve N05 is situated on the side of smaller fatigue lives. 


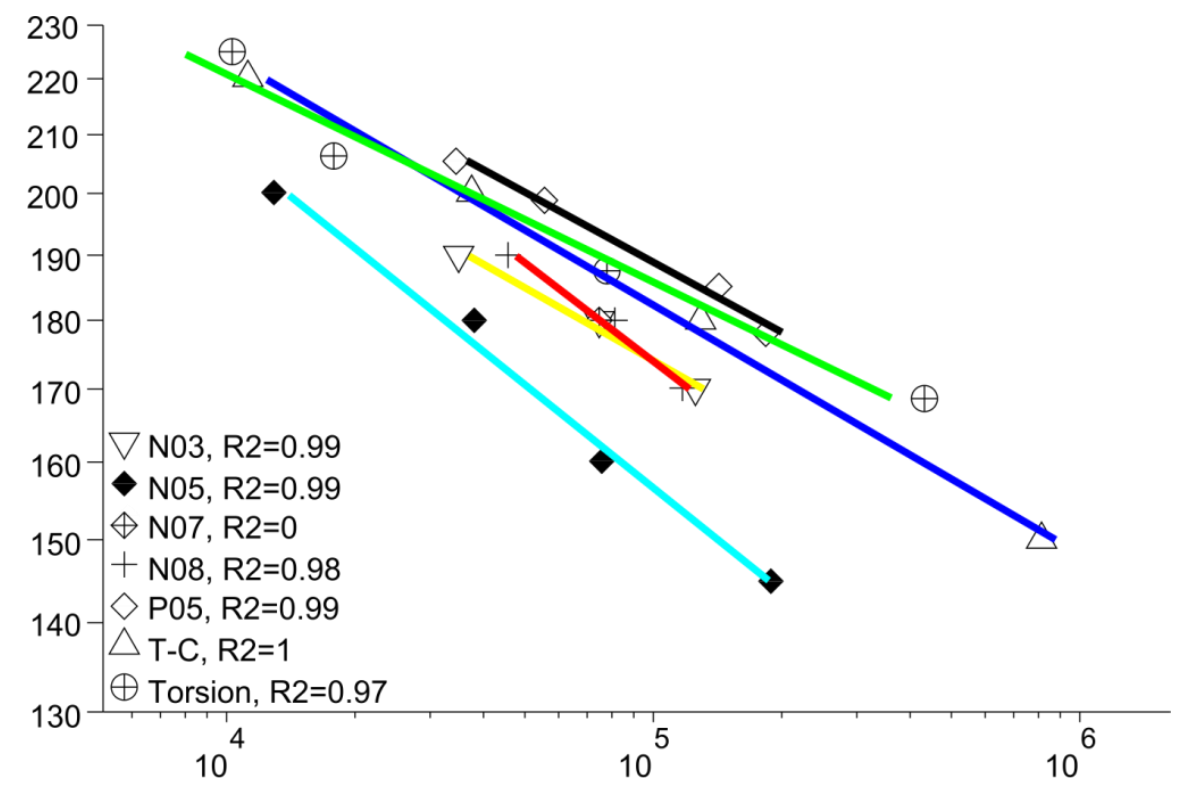

Figure 3. Curves S-N obtained on the basis of equivalent stresses according to Zenner and Liu criterion calculated for each moment of the cycle, for copper CuETP.

The next step of the proposed modification involves taking into consideration a quantity being a measure of non-proportionality of load cycle and the coefficient defining the degree of a material sensitivity to non-proportionality. In the analyzed load case, that is, tension and compression with alternate torsion of sinusoidal courses, phase shifted by $90^{\circ}$, the tangent stress vector draws an ellipse on the plane. The proposed description of such a path of load involves determination of vector $\boldsymbol{\tau}_{\gamma \varphi n}$, normal to shear stress vector with maximum module $\boldsymbol{\tau}_{\boldsymbol{\gamma} \varphi m}$, as is shown in Fig. 4 (a). The larger non-proportionality of load the larger module of vector $\boldsymbol{\tau}_{\boldsymbol{\gamma} \boldsymbol{n} \boldsymbol{n}}$ (Fig. 4 (b)) and for this reason it has been accepted as the quantity to be used as a non-proportionality measure.

(a)

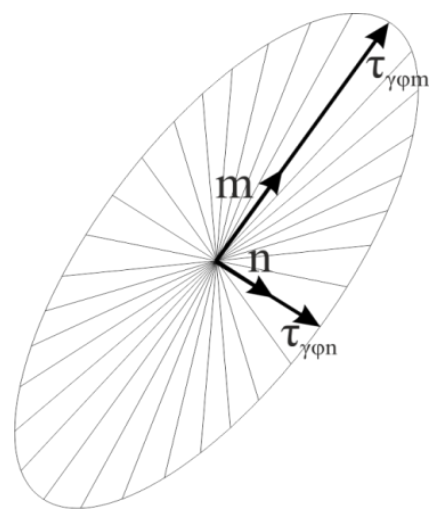

(b)

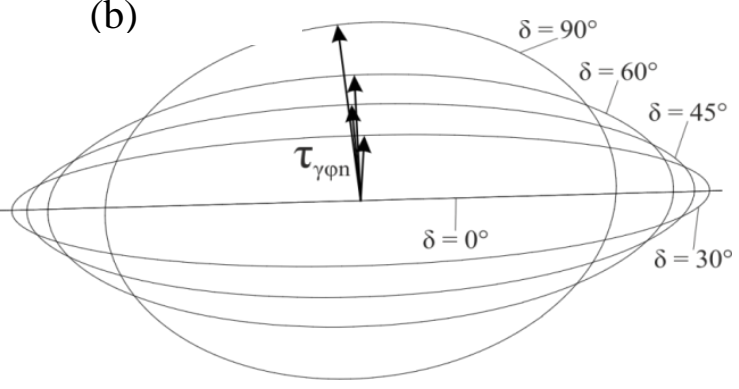

Figure 4. (a) Vectors $\boldsymbol{\tau}_{\boldsymbol{\gamma} \varphi m}$ and $\boldsymbol{\tau}_{\boldsymbol{\gamma} \boldsymbol{} \text { n }}$ describing the load path, (b) paths drawn by shear stress vector for different angles of phase shift $\delta$. 
Vector $\boldsymbol{\tau}_{\boldsymbol{\gamma} \boldsymbol{} \boldsymbol{n}}$ can be determined in the following way: unit vector $\boldsymbol{m}$ has to be found in direction $\boldsymbol{\tau}_{\gamma \varphi m}$, next it needs to be turned by $90^{\circ}$, thus obtaining coordinates of vector $n$ :

$$
\boldsymbol{m}=\frac{\boldsymbol{\tau}_{\gamma \varphi m}}{\left\|\tau_{\gamma \varphi m}\right\|}, \quad \boldsymbol{n}=\boldsymbol{m}\left[\begin{array}{cc}
\cos \frac{\pi}{2} & -\sin \frac{\pi}{2} \\
\sin \frac{\pi}{2} & \cos \frac{\pi}{2}
\end{array}\right] .
$$

Next, cosinuses of the angles between successive positions of vector $\tau_{\gamma \varphi}$ and vector $\boldsymbol{n}$ are to be found, and components $\boldsymbol{\tau}_{\gamma \varphi}$ in direction $\boldsymbol{n}$ are to be determined:

$$
\cos \alpha(t)=\frac{\boldsymbol{\tau}_{\gamma \varphi} \boldsymbol{n}}{\left\|\boldsymbol{\tau}_{\gamma \varphi}\right\|\|\boldsymbol{n}\|}, \quad \boldsymbol{\tau}_{\boldsymbol{\gamma} \boldsymbol{n}}(t)=\boldsymbol{\tau}_{\boldsymbol{\gamma} \boldsymbol{}} \cos \alpha
$$

Vector $\boldsymbol{\tau}_{\gamma \varphi n}$ is a projection of $\boldsymbol{\tau}_{\gamma \varphi}$ onto $n$ with the largest module. A root mean square from the modules has been accepted as the quantity representing non-proportionality measure:

$$
\tau_{\gamma \varphi n R M S}=\sqrt{\frac{15}{8 \pi} \int_{\gamma=0}^{\pi} \int_{\varphi=0}^{2 \pi}\left\|\tau_{\gamma \varphi n}\right\|^{2} \sin \gamma d \gamma d \varphi}
$$

Observing the course of equivalent stress according to Liu and Zenner criterion (Fig. 3), calculated for each moment of the load cycle, it can be observed that for different $\lambda$ and $\sigma_{Z}=$ const, the area under the chart is filled in different ways. In Fig. 5 there is a chart that shows $\tau_{\gamma \varphi n R M S}$ and the difference between the expected value of equivalent stress $\sigma_{\text {exp }}$, established on the basis of experimental fatigue lives, and that $\sigma_{Z, \max (t)}$ calculated in function of $\lambda$.

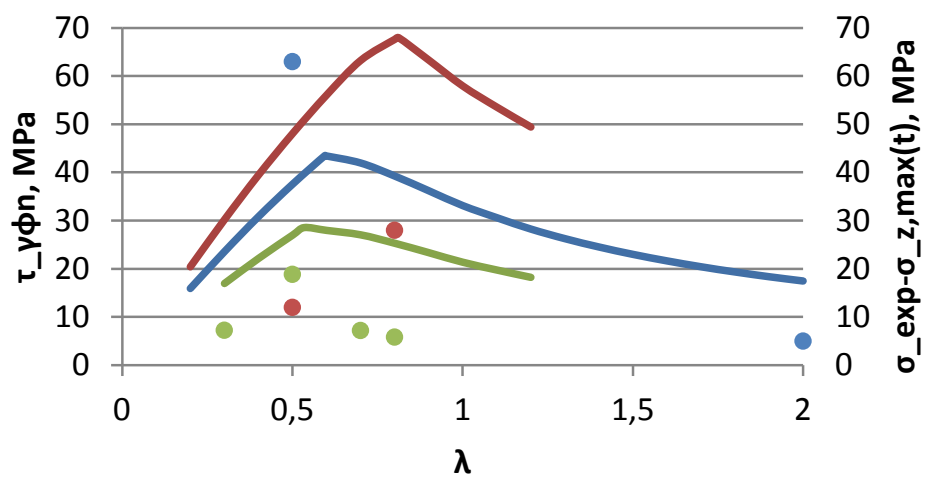

$\tau$ -

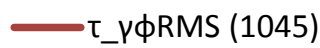

$\tau_{-} \gamma \phi R M S(C U E T P)$

- CuETP o_exp-o_z,max(t)

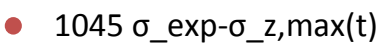

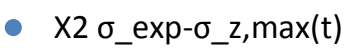

Figure 5. Chart of $\tau_{\gamma \varphi n R M S}$ and the difference between $\sigma_{\text {exp }}$ and $\sigma_{Z, \max (t)}$ in function of $\lambda$. 
On the basis of the above chart it can be observed that $\tau_{\gamma \varphi n R M S}=f(\lambda)$ makes up two intervals: for $\lambda>\tau_{T} / \sigma_{T C}$ and $\lambda<\tau_{T} / \sigma_{T C}$. In these intervals it changes almost in a linear way. The difference between $\sigma_{\text {exp }}-\sigma_{Z, \max (t)}$ changes in a similar way. This allows to state that this is coefficient $\alpha_{\text {mat }}$ that can decide about part $\tau_{\gamma \varphi n R M S}$ which is to be taken into account. According to the proposed modification this coefficient can be defined as a linear function of variable $\tau_{T} / \sigma_{T C}-\lambda$, that is, a distance of the considered ratio $\lambda$ from its critical value (dividing into intervals) for a given material.

Finally, modified form of the criterion can be given by formula:

$$
\sigma_{e q}=\sigma_{z, \max (t)}+\tau_{\gamma \varphi n, R M S} \cdot \alpha_{m a t}
$$

\section{VERIFICATION OF PROPOSED MODIFICATION}

Zenner criterion has been verified for steels 1045 [7], X2CrNiMo17-12-2 [6] and copper CuETP. The verification involves comparing experimental fatigue life $N_{\text {exp }}$ with the calculated $N_{\text {cal }}$. Equivalent stresses have been linked with fatigue lives by means of Basquin equation:

$$
\sigma_{e q}=A N^{B}
$$

Coefficients and equations $\mathrm{A}$ and $\mathrm{B}$ have been determined on the basis of data obtained for tension-compression. Transforming Eq. 14, a dependence is obtained which allows to calculate fatigue lives on the basis of equivalent stresses:

$$
N_{c a l}=\left(\frac{\sigma_{e q}}{A}\right)^{\frac{1}{B}} \text {. }
$$

Experimental fatigue lives have been compared with the calculated ones by means of charts shown in Fig. 6. They provide the basis to state that the proposed modification has improved the consistence of experimental fatigue lives with the calculated ones. 

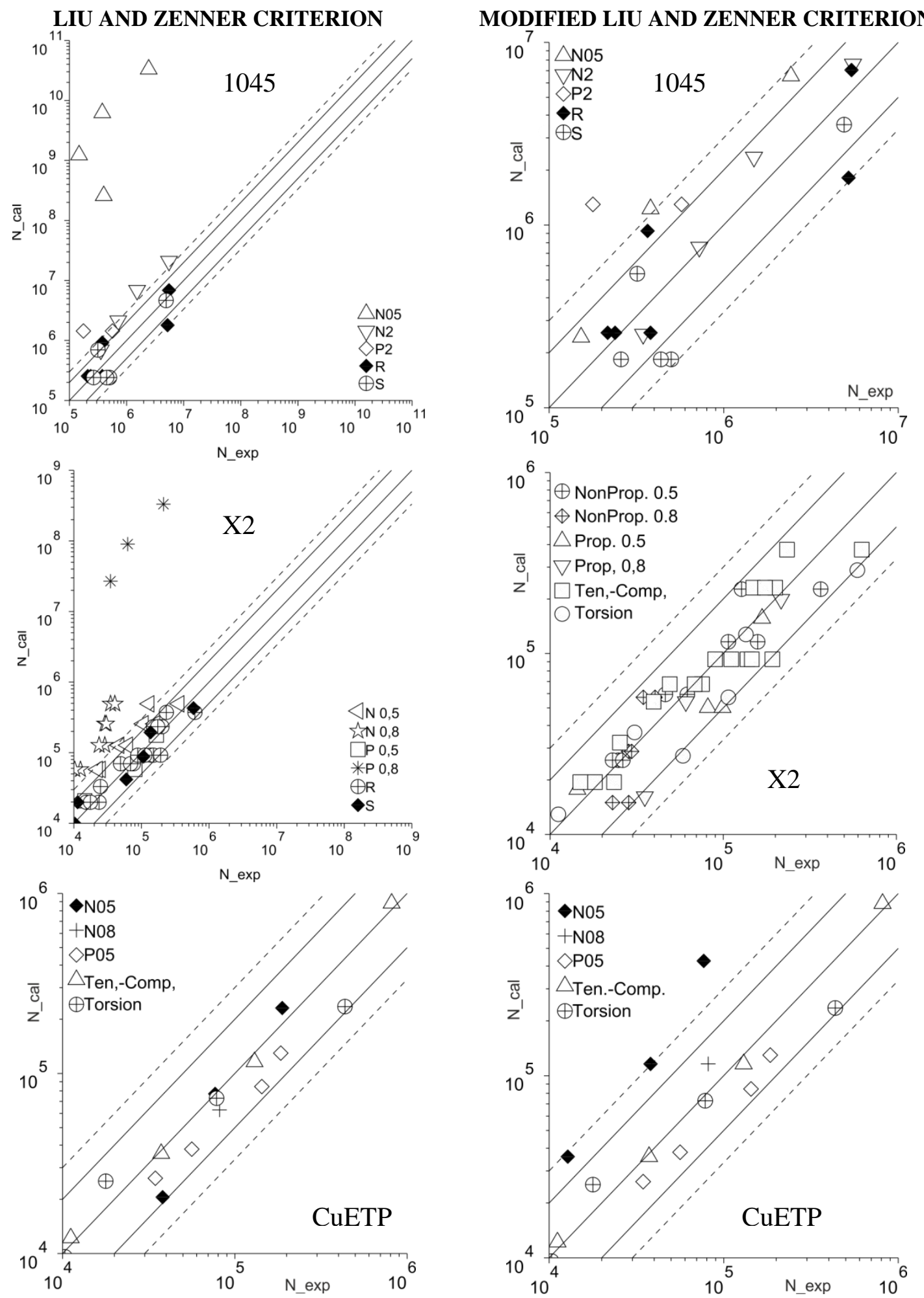

Figure 6. Comparison of experimental and calculated fatigue lives according to Liu and Zenner criterion and to the modified one. 


\section{CONCLUSIONS}

On the basis of the carried out test it can be claimed that:

1. the proposed non-proportionality measure is sensitive to the load path change caused by a shift in the phase between the components,

2. it also allows to account for the impact of tangent to normal ratio $\lambda$ on the nonproportionality degree,

3. the proposed modification of Liu and Zenner criterion enables to count for the material sensitivity to load non-proportionality,

4. in case of materials for which the modification has been performed, the consistence of calculated fatigue lives with the experimental ones has improved.

\section{ACKNOWLEDGEMENTS}

The project has been financed by Polish National Center for Science.

\section{REFERENCES}

1. Zenner H. and Richter I., (1977) Eine Festigkeitshypothese für die Dauerfestigkeit bei beliebigen Beanspruchungskombinationen, Konstruktion, 29, pp. 11-18.

2. Zenner H., Heidenreich R. and Richter I., (1980) Schubspannungsintensitätshypothese - Erweiterung und experimentelle Abstützung einer neuer Festigkeitshypothese für schwingende Beanspruchung, Konstruktion, 32, pp. 143-152.

3. Zenner H., Simbürger A. and Liu J., (2000) On the fatigue limit of ductile metals under complex multiaxial loading, International Journal of Fatigue, 22, pp. 137-145.

4. Papadopoulos I. V. et al., (1997) A comparative study of multiaxial high-cycle fatigue criteria for metals, International Journal of Fatigue, 19, pp. 219-235.

5. Xiao L. and Kuang Z. B., (1996) Biaxial path dependence of macroscopic response and microscopic dislocation substructure in type 302 stainless steel. Acta Materialia 44, pp. 3059-3067.

6. Skibicki D. and Pejkowski Ł., (2012) Integral fatigue criteria evaluation for life estimation under uniaxial, combined proportional and non-proportional loadings, Journal of Theoretical and Applied Mechanics, 50, pp. 1073-1086, Warsaw.

7. Verreman Y. and Guo H., (2007) High-cycle fatigue mechanisms in 1045 steel under non-proportional axial-torsional loading, Fatigue \& Fracture of Engineering Materials \& Structures, 30, pp. 932-946. 